Untersuchungen aus dem chemischen Institut der Universität Strassburg.

\title{
Ueber Umlagerungen bei den ungesättigten Säuren;
} von Rudolph Fittig.

[Vierte Abhandlung.]

\section{Zweibasische ungesättligte Säuren ${ }^{1}$ ).}

Die eigenthümlichen Isomerieverhältnisse zwischen der Fumar- und Maleïnsäure, der Ita-, Citra- und Mesaconsäure haben schon früh die Aufmerksamkeit der Chemiker auf sich gelenkt und sind bis in die allerletzte Zeit der Anlass zu einer grossen Anzahl von Experimentaluntersuchungen gewesen, ohne dass die neueren Arbeiten das Verständniss der bei diesen

1) Von der umfangreichen Untersuchung, über welche ich hier ausfïhrlich berichte, habe ich einige der wichtigeren Thatsachen von Zeit zu Zeit in kurzen, vorläufigen Notizen (Ber. d. deutsch. chem. Ges. 26, 43, 2082; 27, 2680; 29, 1842) mitgetheilt. Die Einzelheiten, soweit sie nicht die von mir allein ausgeführten Versuche betreffen, sind dann mach und nach in Dissertationen publicirt, welche heute dem sich dafïr Interessivenden leicht zugänglich sind, da sie durch regelmässigen Austausch in die Bibliothek der deutschen chemischen Gesellschaft und alle grösseren Universitätsbibliotheken gelangen. In diesen Dissertationen sind dann auch immer die theoretischen Folgerungen gezogen, welche sich aus den bis dahin bekannten Thatsachen ergaben. Meiner Gewohnheit gemäss habe ich mit der ausfïhrlichen Publication gewartet, bis das Ganze mehr abgerundet und einzelne von mir beanstandete Versuchsresultate durch Wiederholung der Versuche auf ihre Richtigkeit geprüft und bestätigt oder berichtigt waren. Das erforderte selbstverständlich viel Zeit. Während dem sind mehrere Publicationen erschienen, welche zu unserer Untersuchung in gewisser Beziehung stehen. Ich citire diese nur und nehme auf sie nur Rücksicht, wenn sie von irgend welchem Einfluss auf unsere Arbeiten waren. Das gilt speciell für einige Publicationen von Stobbe. Wie ich soeben aus einem Berichte in der ChemikerAnnalen der Chemie 304. Bd. 
Verbindungen beobachteten zablreichen Umlagerungen wesentlich gefördert haben. Nachdem unsere Kenntnisse uber das chemische Verhalten der einbasischen ungesättigten Säuren durch die Synthese zahlreicher neuer Glieder von ganz bestimmter Collstitution und das exacte Studium derselben in vieler Beziehung wesentlich erweitert worden sind, hoffte ich auch bei den zweibasischen Säuren zu neuen Gesichtspunkten zu gelangen, wenn ich nicht mehr auf die Anfangsglieder der Reihe allein angewiesen war, sondern über ein grösseres Untersuchungsmaterial disponirte. Die Arbeiten, über welche ich im Folgenden berichte und über deren hauptsächlichste Ergebnisse ich hier zunächst eine kurze, zusammenfassende Darstellung geben will, werden zeigen, wie weit sich diese Erwartung erfült hat.

Das Ausgangsmaterial zu diesen Untersuchungen war verhältnissmässig leicht zu beschaffen. Die früher publicirten Arbeiten von mir und meinen Schülern haben gezeigt, dass die durch Condensation von Aldehyden mit bernsteinsaurem Natrium entstehenden Lactonsäuren (Paraconsäuren) bei ihrer trocknen Destillation immer neben ungesättigten einbasischen Säuren die Anhydride von zwei mit den angewandten Paraconsäuren isomeren zweibasischen Säuren liefern, welche mit der Itaconsäure und Citraconsäure homolog sind. Sie haben aber auch weiter dargethan, dass sich die Umwandlung der Lactonsäuren in die der Itaconsäurereihe angehörenden zweibasischen Säuren noch leichter und vollständiger dadurch ausführen lässt, dass man durch Erwärmen der Paraconsäureester mit Natriumäthylat in

Zeitung ersehe, hat dieser Chemiker auf der diesjälnrigen Naturforscherversammlung im September in einem Vortrage seine Ausichten über die Constitution der neuen, von uns entdeckten und als Aticonsäuren bezeichneten Säuren entwickelt. Diese stimmen, wie es scheint, in einigen wesentlichen Punkten ibberein mit den Schlussfolgerungen, welche ich im Folgenden entwickele and welche schon vor mehreren Jahren in verschiedenen Dissertationen, ganz besonders scharf und logisch in der Anfang 1897 erschienenen von Petkow aus den beobachteten Thatsachen abgeleitet sind. 
alkoholischer Lösung diese in die Natriumsalze der sauren Ester der entsprechenden Itaconsäuren umwandelt und aus diesen durch Verseifung die Säuren bereitet. Diese Methode lässt sich bei allen Paraconsäuren anwenden, auch bei den der aromatischen Gruppe angehörenden, während die erstere Methode auf die aliphatischen Paraconsäuren beschränkt ist.

Wir haben uns bei dieser Untersuchung immer der zweiten Methode bedient und die so als Ausgangsmaterial bereiteten Itaconsäuren durch Erhitzen resp. Destillation in die Anhydride der Citraconsäuren umgewandelt. Aus den Citraconsäuren liessen sich ausserordentlich leicht die Mesaconsäuren gewinnen. Es ist seit lange bekannt, dass die Maleïnsäure bei der Einwirkung ron Brom zum Theil in Fumarsäure übergeht. Wir fanden, dass diese Umlagerung bei der Citraconsäure und allen Homologen derselben stattfindet und dass sie unter geeigneten Versuchsbedingungen das weitaus bequemste Mittel zur Gewinnung der Nesaconsäuren ist. Es kommt darauf an, die Citraconsäuren in einem indifferenten Mittel zu lösen, in welchem sie selbst in der Kälte leicht, die Mesaconsäuren aber wenig oder nicht löslich sind. Als sehr geeignet erwies sich das Chloroform. Nur die gewöhnliche Citraconsäure ist darin recht schwer löslich und bei ihr muss deshalb reiner Aether als Lösungsmittel angewandt und die Lösung mit möglichst viel Chloroform gemischt werden. Setzt man nun zu einer solchen Lösung irgend einer Citraconsäure nur einen Tropfen einer Lösung von Brom in Chloroform, so beginnt im directen Sonnenlichte fast momentan die Abscheidung der entsprechenden Mesaconsäure und in ganz wenigen Minuten ist weitaus die grösste Menge der angewandten Citraconsäure in Mesaconsäure umgewandelt, die nur abfiltrirt und mit Chloroform gewaschen zu werden braucht, um absolut rein erhalten zu werden.

Diese Umwandlung, in der beschriebenen Weise ausgeführt, gehört zu den merkwürdigsten and überraschendsten Reactionen, welche ich kenne. Sie ist aber niemals eine ganz vollständige, denn das freie Brom verschwindet nach einiger Zeit und es 
entstehen ölige, bromhaltige Producte, die gelőst bleiben. Bei einigen Säuren haben wir bis zu $93 \mathrm{pC}$, bei andern $70-80 \mathrm{pC}$. Ausbeute erhalten. Ich weiss nicht, ob die Ursache dieser verschiedenen Ausbeuten von den Versuchsbedingungen: Menge des Lösungsmittels, Intensität des directen Sonnenlichtes u. s. w., die ja nicht immer gleich gehalten werden können, abhängen; auf jeden Fall aber kommt auch die Natur der Säure dabei in Betracht, denn die Dimethylcitraconsäure liefert unter allen Bedingungen eine viel geringere Ausbeute an Mesasäure, als die andern Citrasäuren. Sehr bemerkenswerth ist das Verhalten der mit der Pyrocinchonsäure isomeren Säuren. Da die der Citrareihe angehörende Pyrocinchonsäure nicht existirt, lässt sich bei ihr die Umlagerung nicht ausführen, denn die Anhydride sind überhaupt dazu nicht befähigt. Das Auffällige aber ist, dass hier umgekehrt die der Mesareihe angehörende Dimethylfumarsäure unter den gleichen Versuchsbedingungen theilweise in Pyrocinchonsäureanhydrid zurückverwandelt wird. Das führt fast zu der Vermuthung, als ob auch diese Umlagerung, wie fast alle anderen, ein umkehrbarer und deshalb durch einen Grenzzustand beschränlzter Process ist. Bei der Pyrocinchonsäure kommt in Betracht, dass sie sofort in ihr Anhydrid übergeht und auf dieses Brom nicht einwirkt, während bei den anderen Säuren sich das kaum wird nachweisen lassen, weil die Citrasäuren leichter Brom addiren, als die tibrigen Säuren und es keine Lösungsmittel giebt, in denen die Mesasäuren leicht und die Citrasäuren schwer löslich sind. Die Isomeren der Pyrocinchonsäure zeigen übrigens auch in anderer Hinsicht noch Unterschiede im Verhalten von den ubrigen analog constituirten Säuren. Hier ist die Citrasäure die beständigste, vielleicht eben weil boi ihr sofort die sehr feste Anhydridbildung stattfindet.

Es ist kaum möglich, sich eine Vorstellung zu machen, wie diese ausserst geringen Mengen von Brom wirken. Wenn man die Umwandlung vor seinen Augen verlaufen sieht, denkt man unwillkürlich an eine fermentartige Wirkung des Broms, 
die aber ausserordentlich viel rascher stattfindet, als andere Fermentwirkangen. Dass eine etwaige Bromwasserstoffbildung hier keine Rolle spielen kann, zeigt schon die Thatsache, dass die Umlagerung sofort aufhört, wenn die geringe Menge des zugesetzten Broms durch Nebenreactionen verbraucht ist, dass sie aber durch Zusatz einer neuen, kleinen Menge Broms sofort wieder eingeleitet wird. Aber auch unsere bei der einfachen Citraconsäure ausgeführten Versuche beweisen direct, dass Bromwasserstoff unter sonst gleichen Verbältnissen ohne jede Wirkung ist.

Was die Namen der neuen Säuren betrifft, so balte ich es für pralktisch, sie nicht von der Maleïn- und Fumarsäure, sondern von den Ita-, Citra- und Mesaconsäuren abzuleiten, weil bei den Itaconsäuren es ein niedrigeres Homologon als die gewöhnliche Itaconsäure nicht giebt und die Bezeichnung Aethylparaconsäure, Aethylitaconsäure, Aethylcitraconsäure und Aethylmesaconsüure jedenfalls übersichtlicher ist, als wenn man die isomeren Säuren als Aethylparaconsäure, Aethylitaconsäure, Propylmalë̈nsäure und Propylfumarsäure bezeichnen wollte.

Die isomeren Säuren der drei Reihen lassen sich leicht von einander trennen und in einem Gemenge quantitativ bestimmen. Die Citraconsäuren sind dadurch charakterisirt, dass sie in wässriger Lösung schon bei der Siedetemperatur des Wassers in Anbydride übergehen und diese mit Wasserdämpfen flüchtig sind. Aus einem Gemenge der drei Säuren lassen sich deshalb in allen Fällen die Citraconsäuren durch hinreichend lange Destillation mit Wasser überdestilliren, während die Itaund Mesaconsäuren nicht flüchtig sind und im Destillationsrückstande bleiben. Bei der Destillation mit Wasser findet mit einer Ausnahme, auf die ich gleich zurückkomme, keine Veränderung der drei Säuren statt.

Die Itacon- und Mesaconsäuren lassen sich leicht durch ihre Baryum- oder Calciumsalze trennen, denn die itaconsauren Salze sind in Wasser, zumal in heissem, sehr schwer löslich, während die mesaconsauren Salze so leicht löslich sind, dass 
ihre Lösungen fast immer zur Syrupconsistenz verdunstet werden können, bevor sich etwas abscheidet.

Die Ita- und Citraconsäuren besitzen keinen glatten Schmelzpunkt; bei ihnen fällt die Schmelz- und Zersetzungstemperatur (in Anhydrid und Wasser) zusammen, oder die Zersetzung findet bei noch niedrigerer Temperatur statt. Deshalb wird die Temperatur, bei welcher das Flüssigwerden der Säuren eintritt, durch das schnellere oder langsamere Erwärmen beeinflusst. Die Citraconsäuren schmelzen fast sämmtlich unter $100^{\circ}$ (nur die Phenylcitraconsäure etwas über $100^{\circ}$ ), der Schmelzpunkt der Itaconsäuren liegt beträchtlich höher.

Die Mesaconsäuren haben fast immer den höchsten Schmelzpunkt (nur bei den Isopropylverbindungen liegt der der Itaconsäure einige Grade über dem der Mesaconsäure) und sie unterseheiden sich von den anderen Säuren so wesentlich dadurch, dass bei ihnen die Schmelztemperatur eine constante ist, dabei keine Zersetzung stattfindet und dass sie oberhalb dieser bei vorsichtigem Erhitzen fast ganz unzersetzt sublimiren. Dabei geht nur ein kleiner Theil in das Anhydrid der Citrasäure über. Einmal, bei der Dimethylmesaconsäure, konnten wir auch die Bildung einer geringen Menge des Anhydrids der Itaconsäure nachweisen.

Den Itaconsäuren und den Citraconsäuren entsprechen Anhydride, in welche sie durch Erhitzen verwandelt werden. Bei der Destillation unter gewöhnlichem Luftdruck wandeln sich die Itaconsäureanhydride grösstentheils in die isomeren Citraconsäureanhydride um, weshalb man bei der Destillation der Itaconsäuren immer ein Gemenge beider Anhydride in verschiedenen, von der Art des Erhitzens abhängigen Verhältnissen erhält. Daneben entstehen immer kleine Mengen von gelben, indifferenten Oelen. Bei der Destillation im Vacuum aber verläuft der Process sehr viel glatter und das Destillat besteht fast ganz aus dem Itaconsäureanhydrid. Unsere Versuche aber haben das merkwürdige Resultat ergeben, dass die Umwandlung der beiden Anhydride in einander durch Erwärmen ein umkehrbarer Process 
ist, bei welchem also ein Grenzverhältniss eintritt. Deshalb entsteht in der Hitze niemals uur das eine Anhydrid, sondern immer bilden sich beide nebeneinander. Auch bei der vorsichtigsten Destillation der Itaconsäuren im Vacuum enthält das Product immer einige Procent des Citraconsäureanhydrids, und die Anhydride der Itaconsäuren lassen sich aus diesem Grunde durch Destillation der Săuren nur rein gewinnen, wenn sie (wie bei der Dimethyl- und Phenylitaconsäure) feste, krystallinische Körper sind, die man mit Lösungsmitteln und durch Umkrystallisiren von der geringen Menge des Citraconsäureanhydrids treunen kann; sind sie aber flüssig, oder durch das beigemengte Citraconsäureanhydrid flüssig gehalten, so gelingt die Isolirung der reinen Anlydride nicht. Einen directen Beweis, dass die Sache sich so verhält, lieferte uns die Untersuchung der Phenylitaconsäure. Hier lassen sich die Anhydride nicht durch Destillation der Säure bereiten, weil dabei auch im Vacuum tiefgreifende Zersetzungen stattinden, aber schon beim blossen Schmelzen im Vacuum findet die Anhydridbildung statt und schon bei dieser verhältnissmässig niederen Temperatur bilden sich neben dem Itaconsäureanhydrid immer einige Procent des isomeren Citraconsäureanhydrids, welches mit Lösungsmitteln entfernt werden kann. Das so gereinigte Itaconsäureanhydrid braucht nur im Vacuum oder unter Luftdruck wieder geschmolzen und dann rasch abgekühlt zu werden, so enthält es eine neue Menge des Citraconsäureanhydrids und so fort. Auf diese Weise nur liess sich die Phenylcitraconsäure, welche zu unserer Untersuchung diente, gewinnen. Das reine Phenylcitraconsäureanhydrid aber lagert sich beim Erwärmen über seinen Schmelzpunkt, ja langsam schon unter $100^{\circ}$ (in einem Bade von siedendem Alkohol) zum Theil wieder in das Anhydrid der Itaconsäure um.

Dieses Verhalten der Anhydride giebt nun den Schlüssel zum Verständniss einer lange bekannten Reaction. Die gewöhnliche Citraconsäure geht bekanntlich beim Erhitzen ihrer wässrigen Lösung in zugeschmolzenen Röhren auf $130-150^{\circ}$ 
in Itaconsäure über und diese Umlagerung wird ja allgemein zur Gewinnung der Itaconsäure benutzt. Nach unseren Versuchen findet unter denselben Bedingungen die Umwandlung aller der Citrareihe angehörigen Säuren in die isomeren Itasäuren statt, bei $130^{\circ}$ langsam, über $150^{\circ}$ rascher, und bei genügend langem Erhitzen ist sie eine vollständige. Nebenproducte entstehen dabei nicht. Bei den der aliphatischen Gruppe angebörenden Citraconsäuren erfolgt diese Umlagerung bei $100^{\circ}$ noch nicht, aber sie spalten sich bei dieser Temperatur in ihrer wässrigen Lösung schon in Anhydride und Wasser, und die Thatsache, dass alle Citraconsäuren mit Wasser überdestillirt werden können, beruht darauf, dass nicht sie, sondern ihre Anbydride mit Wasserdämpfen flüchtig sind. Man kann das scharf beobachten, denn bei der Destillation der wässrigen Lösungen dieser so leicht löslichen Säuren gehen mit den Wasserdämpfen fortdauernd Oeltröpfchen über, die sich zum Theile erst in der Vorlage wieder allmählich klar auflösen. Besonders instructiv ist hier wieder das Verhalten der Phonylcitraconsäure, deren Anhydrid fest ist und sich viel langsamer mit Wasser verbindet. Bei der Destillation der verdünnten Lösung dieser Säure wird nun ein anfangs ganz neutral reagirendes Destillat erhalten, in welchem das feste Anhydrid, welches sich schon im Kühler abscheidet, suspendirt ist. Bei Temperaturen über $100^{\circ}$ aber beginnt die theilweise Umlagerung der Citraconsäureanhydride in die isomeren Itaconsäureanbydride. Ein Gleichgewichtszustand ist bei Gegenwart von Wasser nicht mehr möglich, da die Itaconsäureanhydride mit dem Wasser sofort in die unter den Versuchsbedingungen beständigen Itaconsäuren übergehen. Deshalb muss die Umwandlung allmählich eine vollständige werden.

Wenn diese Erklärung des Vorganges die richtige ist, musste die Phenylcitraconsäure im Gegensatze zu den übrigen Citraconsäuren schon beim Kochen mit Wasser in die Phenylitaconsäure übergehen, da ja ihr Anhydrid sich langsam und zu einem geringen Theile schon unter $100^{\circ}$ in das isomere 
Itaconsäureanhydrid umlagert. Der Versuch zeigte, dass das in der That der Fall ist.

Durch Erhitzen mit Chlor- oder Bromwasserstoff und durch geeignete Behandlung mit Schwefelsäure werden die Itaconsäuren in die Paraconsäuren, aus welchen sie dargestellt waren, zurückverwandelt. Nur eine Säure, die Isopropylitaconsäure, macht hier eine sehr merkwürdige Ausnahme von der sonst allgemeinen Regel, indem sie auf beide Weisen nicht in die gewöhnliche Isopropylparaconsäure, sondern in eine damit isomere einbasische Lactonsäure übergeführt wird. Die Umwandlung erfolgt hier mit besonderer Leichtigkeit und mit nahezu quantitativer Ausbeute. Wir haben uns eingehend mit dieser neuen Lactonsäure beschäftigt und mit Sicherheit festgestellt, dass sie nach der Formel<smiles>CC1(C)CC(CC(=O)O)C(=O)O1</smiles>

constituirt ist, denn sie bildet sich auch bei der Oxydation der Isobutylbernsteinsäure<smiles>CC(C)CC(CC(=O)O)C(=O)O</smiles>

welche wir zu dem Zwecke synthetisch dargestellt baben, nach der Methode von Rich. Meyer durch Oxydation des Wasserstoffs am tertiär gebundenen Kohlenstoffatom zu Hydroxyl und Schliessung des Lactonringes.

Der glatte Uebergang der Isopropylitaconsäure in diese Säure, also von<smiles>CC(C)C(CC(=O)O)C(=O)O</smiles>

ist schwer verständlich, allein unsere Versuche zeigen, dass auch die gewöhnliche Isopropylparaconsäure unter den gleichen Versuchsbedingungen fast glatt in die isomere Lactonsäure uber- 
geht. Wenn also die Isopropylitaconsäure zunächst wie die anderen Itaconsäuren in Isopropylparaconsäure übergeführt wird, so muss doch statt ihrer die isomere Säure erhalten werden. $\mathrm{Ob}$ aber immer eine vorherige Bildung von Isopropylparaconsäure stattfindet, ist nach unseren Versuchen recht zweifelhaft, denn die isomere Lactonsäure entsteht schon in nambafter Menge, wenn man zu einer heissen Lösung der Itaconsäure in überschüssiger Natronlauge rasch concentrirte Salzsäure setzt, während bei einem mit der Isopropylparaconsäure ebenso ausgefuhrten Versuche diese ganz unverändert blieb. Uebrigens ist auch die Umwandlung der Isopropylparaconsäure in die isomere Säure kein einfacher, leicht verständlicher Process, aber wenn man von der Lactonbindung einen Augenblick absieht und die Constitution der beiden Säuren mit geöffnetem Ringe
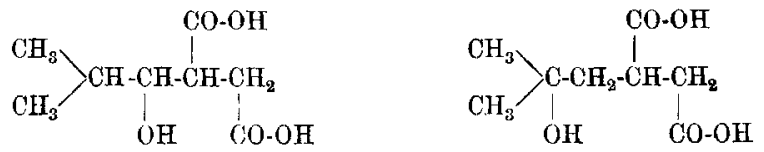

mit einander vergleicht, so sieht man, dass die Umlagerung auf einer Verschiebung des Hydroxyls basirt, welche nicht ohne Analogie ist. Die Umwandlung des Isobutylalkohols in den tertiären Alkohol<smiles>CC(C)CC(C)C</smiles>

durch Erhitzen mit concentrirter Salzsäure ist der einfachste derartige Fall.

Die neue Lactonsäure gehört einer den gewöhnlichen Paraconsäuren parallel laufenden Reihe von Lactonsäuren an, bei denen statt des endständigen, das in der Mitte der Kette befindliche Carboxyl zur Herstellung des Lactonringes dient. Ich bezeichne diese Lactonsäuren, von welchen wir im Laufe unserer Untersuchung noch andere Glieder kennen gelernt haben, auf die ich gleich zuräcklkommen werde, als Isoparaconsäuren. Von den Paraconsäuren unterscheiden sich diese isomeren Säuren im 
chemischen Verhalten dadurch wesentlich, dass der Lactonring bei ihnen sehr viel fester ist und es langen Kochens mit starken Basen bedarf, um die Salze der entsprechenden zweibasischen Säuren zu erhalten. Ein anderer Unterschied - wenn es gestattet ist, aus dem Verhalten der genauer untersuchten Isopropylisoparaconsäure einen Rückschluss auf das der homologen Säuren zu machen - ist der, dass sie fast ohne Zersetzung destillirbar sind und erst bei längerem Erhitzen auf ihren Siedepunkt theilweise in das Anhydrid der isomeren Citraconsäure übergehen, ohne wie die Paraconsäuren ungesättigte einbasische Säuren zu bilden. Auch bei der Einwirkung von Natriumäthylat auf ihre Ester gehen sie nicht in zweibasische, den Itaconsäuren entsprechende, sondern in complicirtere, durch Wasserabspaltung entstandene Säuren über.

Wie die Maleïnsäure und Fumarsäure mit Natriumamalgam in die gleiche Bernsteinsäure, die Ita-, Citra- und Mesaconsäure in die gleiche Brenzweinsäure übergehen, so haben wir immer aus den drei isomeren der Ita-, Citra- und Mesareihe angehörenden Säuren die gleiche substituirte Bernsteinsäure erhalten. Aber die drei Klassen von Säuren zeigen bei der Reduction ganz erhebliche Unterschiede, denn während die Citraconsäuren sich sehr leicht mit Wasserstoff verbinden und aus den Mesasäuren, wenn auch nicht ganz so leicht, doch ohne erbebliche Schwierigkeiten, sich ebenfalls die theoretische Ausbeute an den substituirten Bernsteinsäuren gewinnen lässt, setzen die Itaconsäuren der Reduction einen ganz ausserordentlich grossen Widerstand entgegen. Darin liegt die Ursache meiner früheren nicht ganz exacten Angabe, dass die Teraconsäure und die Isobutylitaconsäure sich mit Natriumamalgam überbaupt nicht reduciren lassen. Jetzt ist es uns durch wochenlange Behandlung mit sehr grossen Mengen Amalgams in heisser, stets neutral oder schwach sauer gehaltener Lösung gelungen, diese und alle anderen Itaconsäuren in die entsprechenden Bernsteinsäuren überzuführen, aber nic haben wir auch nur annähernd theoretische Ausbeuten davon erbalten und stets traten dabei stark 
riechende, flüchtige Nebenproducte auf, über deren Natur wir indess nichts aussagen können.

Diese und alle anderen Resultate unserer Arbeit stimmen gut mit der bisherigen Annahme überein, dass den Itaconsäuren und Citraconsäuren die beiden verschiedenen Constitutionsformeln

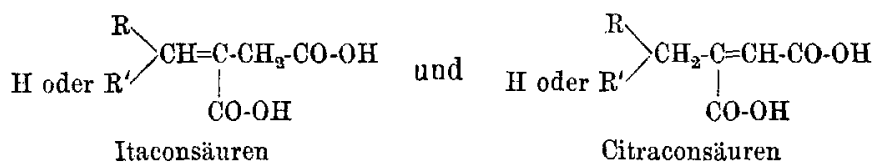

zukommen. Auf das Isomerieverhältniss zwischen den Citraund Mesaconsäuren haben die neu aufgefundenen Thatsachen kein wesentlich neues Licht geworfen.

Von den mannigfaltigen Umlagerungen bei diesen isomeren Säuren haben uns besonders auch die interessirt, welche beim Kochen mit Natronlauge eintreten. Kurz zusammengefasst ist das Resultat unserer Versuche, dass bei ihnen, ebenso wie bei den früher untersuchten einbasischen ungesättigten Säuren eine Verschiebung der Doppelbindung stattfindet, dass aber ähnlich, wie bei jenen, fast niemals eine vollständige Umlagerung stattfindet, sondern der Umlagerungsprocess ein umkehrbarer ist und deshalb sich zwischen der angewandten Säure und den aus ihr entstehenden isomeren Säuren ein bestimmter Gleichgewichtszustand herstellt. Besonders lehrreich sind hier die quantitativ verfolgten Versuche bei der Phenylitaconsäure und ihren Isomeren. Hier finden die Umlagerungen am leichtesten statt, die Grenze derselben wird schon nach verhältnissmässig kurzer Zeit erreicht, und so konnten wir-hier den exacten Nachweis liefern, dass unter gleichen Versuchsbedingungen die qualitative und quantitative Zusammensetzung der Producte ganz unabhängig davon ist, welche der isomeren Säuren dem Einfluss der siedenden Natronlauge unterworfen wird.

Am leichtesten lassen sich die Citraconsiuren umlagern. Sie gehen dabei in die Itaconsäuren über und geben dann weiter die Umlagerungsproducte dieser. Bei ihnen scheint die Umlagerung eine vollständige zu sein. Bei der Phenylcitracon- 
säure enthielt das Endproduct keine Spur von unveränderter Säure mehr, bei den aliphatischen Citraconsäuren ist das Kochen meistens nicht so lange fortgesetzt, bis die letzten Mengen umgewandelt waren, aber bei der Einwirkung der Natronlauge auf die Ita - und Mesaconsäuren haben wir mit ganz vereinzelten Ausnahmen niemals die Bildung der Citraconsäuren wahrnehmen können. Eine solche Ausnahme bilden wieder die aus der Pyrocinchonsäure entstehende Methylita- und -mesaconsäure, welche beim Kochen mit Natronlauge theilweise in Pyrocinchonsäure zurückverwandelt werden. Ausserdem haben wir nur noch aus der Dimethylitaconsäure eine sehr geringe Menge von Dimethylcitraconsäure erbalten und einmal, als wir die nicht substituirte Itaconsäure anstatt mit Natronlauge mit sehr concentrirter Kalilauge gekocht hatten, die Bildung einer geringen Menge Citraconsäure constatiren können.

Die Mesaconsäuren werden am schwierigsten umgelagert. Bei Anwendung von zehnprocentiger Natronlauge konnte in den meisten Fällen auch nach sehr langem Kochen nur der Beginn einer Umlagerung constatirt werden, durch 20 procentige aber wurden sie ebenfalls in die Itaconsäuren übergeführt.

Am interessantesten ist das Verhalten der Itaconsäuren. Die nichtsubstituirte Itaconsäure geht beim Kochen mit Natronlauge leicht und grossentheils (über $70 \mathrm{pC}$ ) in Mesaconsäure über. Da nun die Citraconsäure, wie wir nachweisen konnten, wie alle homologen Säuren, zunächst in Itaconsäure übergeht, die dann weiter grösstentheils in Mesaconsäure verwandelt wird, und da ferner die Mesaconsäure selbst sich theilweise zu Itaconsäure umlagert, so muss das Endproduct, von welcher der drei Säuren man auch ausgeht, immer aus einem Gemenge von Itaconsäure und Mesaconsäure bestehen, in welchem die letztere Säure sehr vorherrscht. Das zeigen unsere Versuche klar. Dieses Verhalten der drei Säuren liefert nun eine Darstellungsmethode für die Mesaconsäure, welche einfacher und ergiebiger, als alle anderen bekannten Methoden ist. Man braucht nur das Destillationsproduct der Citraconsäure direct mit zehn- 
procentiger Natronlauge etwa sechs Stunden zu kochen. Bei späteren Darstellungen der Mesaconsäure haben wir diese Methode mit Vortheil angewandt.

Dieses Verhalten der einfachen, nichtsubstituirten Säuren findet sich in dem Maasse aber bei keiner der homologen Säuren wieder. Nur bei der aus Pyrocinchonsäure gewonnenen $\beta$-Methylitaconsulure haben wir noch ansehnliche Mengen (28 pC.) und bei der Phenylitaconsäure geringe Mengen (5 pC.) der entsprechenden Mesaconsäuren aus dem Umlagerungsproducte isoliren können. Bei den anderen untersuchten Itaconsäuren liess sich die Bildung von Mesaconsäuren überhaupt nicht nachweisen.

Allein auch dicse Itaconsäuren sind keineswegs beständig gegen kochende Natronlauge, sondern sie werden dadurch zu neuen isomeren Säuren umgelagert, welche ich als Aticonsäuren bezeichne. Diese sind ebenfalls zweibasische ungesättigte Säuren und bilden eine vierte, den Ita-, Citra- und Mesaconsäuren parallel laufende Reihe. Von den Itaconsäuren gehen beim Kochen mit Natronlange durchschnittlich $15 \mathrm{pC}$. in die neuen Säuren über, und da diese, wenn sie in reinem Zustande mit Natronlauge gekocht werden, wieder grösstentheils in die Itaconsäuren zurückverwandelt werden, so stellt sich auch bei dieser Umlagerung ein Gleichgewichtsverhältniss her, welches bei fortgesetztem Kochen nicht mehr verändert wird.

Von diesen neuen Säuren haben wir die

Dimethylaticonsäure,

Isobutylaticonsäure,

Hexylaticonsäure

und Phenylaticonsäure

ganz rein dargestellt und eingehend studirt.

Von den darauf untersuchten Itaconsäuren lieferten nur die einfache nichtsubstituirte Itaconsäure, die $\beta$-Methylitaconsäure aus der Pyrocinchonsäure und. auffälliger Weise die, wie oben erwähnt, zu anderen Umlagerungen so sehr disponirte Isopropylitaconsäure keine Aticonsäuren, wăhrend wir bei der 
normalen Propylitaconsäure die Bildung der Aticonsäure nachzuweisen und diese in kleiner Menge auch rein darzustellen vermochten.

Das Studium der neuen Säuren wurde dadurch ausserordentlich erschwert, dass zur Gewinnung ausreichender Quantitäten zunächst die recht mühsame Herstellung grosser Mengen der Itaconsäuren erforderlich war und dass diese bei jedesmaligem Kochen mit Natronlauge sich nur in so verhältnissmässig geringem Umfange umlagerten. Noch grössere Schwierigkeiten aber bot die Reindarstellung der neuen Säuren. So leicht die Trennung von der Hauptmenge der unverändert gebliebenen Itaconsäuren ist, so mühsam und zeitraubend war die Entfernung der letzten Mengen derselben, was um so auffälliger ist, da doch die Löslichkeitsverhältnisse der Itaconsäuren so sehr verschieden von denen der Aticonsäuren sind; aber im Gemenge mit den Aticonsäuren ändern die Itaconsäuren vollständig ihre Eigenschaften, sie werden wie jene leicht löslich, selbst in Lösungsmitteln, in welchen sie für sich nahezu gauz unlöslich sind, ja durch ihre Gegenwart vergrössern sie sogar oft noch die Löslichkeit der Aticonsäuren. Ich habe Aehnliches bei anderen Körpern in auch nur annähernd gleichem Maasse während meiner vieljährigen Praxis niemals beobachtet. Für die exacte Untersuchung war aber die vollkommene Reindarstellung der neuen Säuren Bedingung und mit viel Geduld und Ausdauer baben wir diese denn auch erreicht.

In ihren physikalischen Eigenschaften unterscheiden sich die Aticonsäuren von den Itaconsäuren dadurch, dass sie in Wasser und ganz besonders in Aether, aber auch in anderen indifferenten Lösungsmitteln viel leichter löslich sind und ihr Schmelzpunkt bedeutend niedriger liegt. Anscheinend schmelzen sie ohme Veränderung, aber bei den über $100^{\circ}$ schmelzenden findet wahrscheinlich beim Schmelzen doch schon eine beginnende Anhydridbildung statt, denn wenn man mit der erkalteten Probe die Schmelzpunktsbestimmung wiederholt, so wird sie in der Regel schon bei einer etwas niedrigeren Temperatur als das erste Mal flüssig. 
In der That bilden die Aticonsäuren, wio die Ita - und Citraconsäuren selbständige Anhydride, aus denen sie durch Behandeln mit Wasser regenerirt werden. Wir haben von der Dimethyl- und der Phenylaticonsäure diese Anhydride dargestellt und untersucht.

Sehr auffällig ist es, dass diese neuen Säuren in gewisser Beziehung sehr unbeständig sind. Sie werden in wässriger Lösung schon bei Wasserbadtemperatur langsam zersetzt. Die Dimethylaticonsäure, bei welcher wir diese Zersetzung genau studirt haben, war nach viertägigem Erwärmen ihrer wässrigen Lösung im siedenden Wasserbade nahezu vollständig zersetzt. Als Hauptproduct war Isocaprolacton, daneben Terebinsäure und in weit geringerer Menge die Itaconsäure (Teraconsäure) gebildet. Ich hielt es für wahrscheinlich, dass bei dieser eigenthümlichen Zersetzung die Aticonsäure zunächst durch Anlagerung von Wasser an die doppelt gebundenen Kohlenstoffatome und Schliessung des Lactonringes in Terebinsäure übergehe und dass das Isocaprolacton erst aus dieser durch Abspaltung von Kohlensäure entstehe, allein als wir die Terebinsäure in gleich concentrirter Lösung genau in der gleichen Weise und eben so lange erwärmten, blieb sie fast vollständig unverändert und es entstand höchstens eine Spur von Isocaprolacton. Das führt zu der Annahme, dass die Abspaltung von Kohlensäure gleichzeitig mit der Lösung der doppelten Bindung stattfinde und durch diese bedingt sei.

Beim Erwärmen mit verdünnter Schwefelsäure gehen die Aticonsäuren, wie die Itaconsäuren, in Lactonsäuren über, aber während aus der Dimethylaticonsäure glatt und mit theoretischer Ausbeute Terebinsäure entsteht und auch die Phenylaticonsäure die gewöhnliche Phenylparaconsäure liefert, bilden sich aus der Isobutyl- und wahrscheinlich auch aus der Hexylaticonsäure die bereits obell erwähnten Isoparaconsäuren.

Die Aufklärung der Constitution der Aticonsäuren war von hohem wissenschaftlichem Interesse. Es waren zwei Fälle möglich, nämlich : 
1) Die Aticonsäuren sind mit den Itaconsäuren structuridentisch und stehen zu letzteren in dem gleichen, als stereoisomer bezeichneten Verhältnisse, wie die Citraconsäuren zu den Mesaconsäuren, oder

2) die beiden Säurereihen sind structurisomer und die Aticonsäuren durch Verschiebung der doppelten Bindung im Molekül entstanden, wie es die Formeln<smiles>[R]CC(C(=O)O)=C(CC(=O)O)C(=O)O</smiles>

Itaconsäuren<smiles>[R]C=CC(CC(=O)O)C(=O)O</smiles>

Aticonsäuren

veranschaulichen. Das sehr Interessante nun ist, dass keine dieser beiden Annahmen bei allen von uns untersuchten Aticonsäuren anwendbar ist, denn nach dem heutigen Stande der Stereoisomerie-Frage sind bei einer Săure von der Zusammensetzung der Dimethylitaconsäure<smiles>CC(C)=C(CC(=O)O)C(=O)O</smiles>

stereoisomere Modificationen nicht möglich. Danach müsste man also die zweite Annahme für wahrscheinlicher halten, aber diese ist wieder bei der Phenylita- und -aticonsäure unzulässig, denn bei einer Säure<smiles>O=C(O)CC(C(=O)O)c1ccccc1</smiles>

macht der Benzolrest eine solche Verschiebung der doppelten Bindung unmöglich.

Die Resultate unserer Untersuchungen führen nun für die aliphatischen Aticonsüuren fast zwingend zu der zweiten Annahme, sie lassen sich uberhaupt nur erklären, wenn den Aticonsäuren die obige structurisomere Constitution zukommt.

Beweisend ist das Verhalten der gebromten Derivate. In indifferenten Lösungsmitteln addiren die Aticonsäuren leicht zwei Atome Brom, aber die so gebildeten Dibromide sind sehr unbeständig - nur bei der Isobutylaticonsäure ist es uns ge- 
lungen, das Dibromid in reinem Zustande zu isoliren -, sie gehen unter Bromwasserstoffabspaltung in Bromlactonsäuren über, welche als Substitutionsproducte der bereits erwähnten Isoparaconsäuren aufzufassen sind, denn durch Wasserstoff werden sie in diese umgewandelt. Nur die aus der Dimethylaticonsäure entstehende Bromisoterebinsäure macht hier cine äusserst seltsame Ausnahme. Sie geht nämlich bei der Einwirkung von Natriumamalgam auf ihre stets schwach sauer gehaltene kalte Lösung glatt und ganz quantitativ in Dimethylaticonsäure über, während in alkalischer Lösung ausser Dimethylaticonsäure nur noch das Product der Einwirkung von Natronlauge auf die gebromte Säure (Isoheptodilacton) als Nebenproduct entsteht. Danach ist man versucht, diese gebromtc Säure für ein Bromsubstitutionsproduct der Aticonsäure zu halten, allein das ist unzulässig, weil sie einbasisch ist und ihre Umwandlungsproducte beweisen, dass sie, wie die gebromten Süuren aus den anderen Aticonsäuren, eine Bromlactonsäure ist. Die Rückbildung der Aticonsäure daraus, welche durch oft wiederholte Versuche zweifellos festgestellt ist, bleibt einstweilen eine der räthselbaften Reactionen, deren wir melrere bei dem Studium der Aticonsäuren beobachtet haben.

Die Bromlactonsäuren gehen beim Stehen in kalter alkalischer Lösung und nachherigem Ansäuern, oder beim Kochen mit Wasser in feste, gut krystallisirende, neutrale Dilactone über. Daraus folgt, dass das Bromatom in den Bromlactonsäuren zu dem noch vorhandenen Carboxyl in der $\gamma$-Stellung sich befindet und die Reaction nach den Gleichungen 


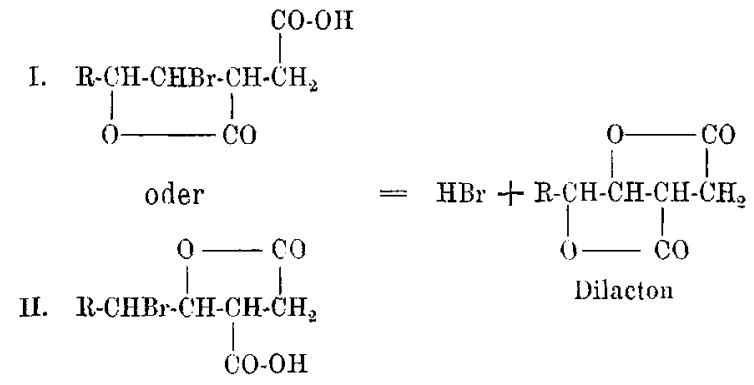

Bromlactonsäure

verlaufen ist. Bromlactonsäuren von der einen oder anderen Constitution aber können nur aus ungesättigten Säuren von der Constitution

$$
\begin{gathered}
\mathrm{R}-\mathrm{CH}=\mathrm{CH}-\mathrm{CH}-\mathrm{CH}_{2}-\mathrm{CO}-\mathrm{OH} \\
\text { CO-OH }
\end{gathered}
$$

entstehen und die Bildung der Dilactone durch Bromanlagerung und Bromwasserstoffabspaltung würde völlig unerklärlich sein, wenn in den ungesättigten Säuren nicht jedes der beiden doppelt gebundenen Kohlenstoffatome zu einem der beiden Carboxyle in $\gamma$-Stellung stände.

Danach ergeben sich für die drei untersuchten aliphatischen Aticonsäuren die folgenden Constitutionsformeln:<smiles>CC(C)C(CC(=O)O)C(=O)O</smiles>

Dimethylaticonsäure<smiles>CC(C)C=CC(CC(=O)O)C(=O)O</smiles>

Isobutylaticonsäure<smiles>CCCCCCCC(CCCC)C(=O)O</smiles>

Hexylaticonsäure.

Die Bromlactonsäuren aus der Isobutyl- und der Hexylaticonsäure müssen nach der ersten der beiden obigen Formeln 
constituirt sein, da sie mit Wasserstoff nicht die gewöhnlichen Paraconsäuren, sondern die isomeren Isoparaconsäuren geben.

Auch für die aus der Dimethylaticonsäure gebildete Bromlactonsäure, Bromisoterebinsäure, ist, wie die gleich näher zu besprechenden Umwandlungsproducte beweisen, von den beiden möglichen Formeln
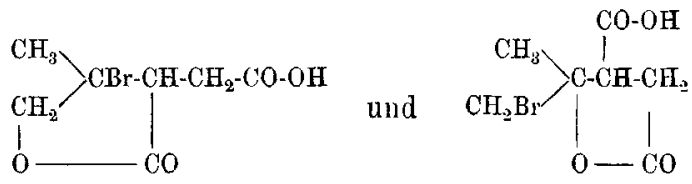

nur die erstere zulässig.

Die Dilactone sind indess nicht die einzigen Zersetzungsproducte der Bromisoparaconsäuren; sic wurden beim Kochen der drei Bromlactonsäuren mit Wasser sogar nur in geringer Ausbeute erhalten und die daneben entstehenden Verbindungen sind bei den drei Säuren nicht ganz gleichartig.

Die Bromisoterebinsäure giebt neben dem Dilacton in kalter alkalischer Lösung als untergeordnetes Nebenproduct, beim Kochen mit Wasser aber als Hauptproduct eine prächtig krystallisirende Säure $\mathrm{C}_{7} \mathrm{H}_{10} \mathrm{O}_{5}$, welche $\mathrm{ich}$, da sie isomer mit der von $\operatorname{Roser}^{2}$ ) aus der Teraconsäure erhaltenen Oxyterebinsäure ist, als Oxyisoterebinsäure bezeichne. Sie ist eine einbasische Lactonsäure mit sehr fest gebundenem Lactonring, dessen Aufspaltung erst bei langem Kochen mit starken Basen stattfindet. Dann aber liefert sie Salze, welche in Zusammensetzung und Eigenschaften mit den aus dem Dilacton bereiteten übereinstimmen.

Diese Oxyisoterebinsäure liess sich auf keine Weise in das Dilacton umwandeln und es gelang auch nicht, umgekehrt das Dilacton in die Säure überzuführen. Danach halte ich es für sehr wahrscheinlich, dass die Oxyisoterebinsäure nach der Formel

2) Diese Annalen $\mathbf{2 2 0}, 263$. 


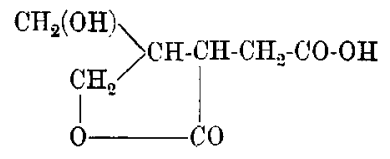

constituirt ist. Die Bildung einer solchen Säure ist etwas überraschend, aber lässt sich doch leicht verstehen. Zweifellos tritt das Bromatom in kalter alkalischer Lösung der Säure in anderer Weise aus, als beim Kochen mit Wasser. Im ersteren Falle, wo der Wasserstoff des Carboxyls durch Natrium ersetzt ist, findet, wie in allen analogen Fällen, der Schluss des Lactonringes unter Austritt von Bromnatrium statt und diese Reaction wird zur Hauptreaction. Beim Kochen mit Wasser aber findet die Lactonbildung nur in sehr untergeordnetem Maasse statt, das Bromatom spaltet sich also mit einem anderen Wasserstoffatom als Bromwasserstoff $a b$. Geschieht dieses nun mit einem Wasserstoffatom des Methyls, so entsteht zunächst eine ungesättigte Lactonsäure:

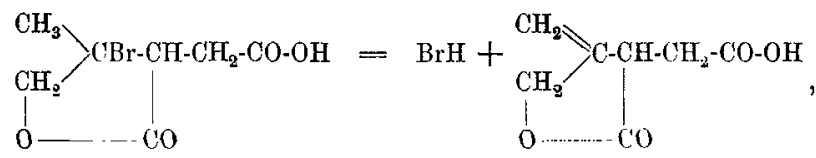

aus welcher durch Anlagerung von Wasser $(\mathrm{H}+\mathrm{OH})$ an die doppelt gebundenen Kohlenstoffatome eine Oxyisoterebinsäure von der obigen Constitution gebildet werden kann.

Mit dieser Formel steht die grosse Beständigkeit der Säure, ihre Unfähigkeit, in das Dilacton überzugehen und aus ihm zu entstehen und ihr ganzes übriges Verhalten im Einklang. Nur eine Beobachtung scheint dem zu widersprechen, die nämlich, dass die Säure beim Kochen mit Basen dieselben Salze wie das Dilacton giebt. Ich lege darauf nur geringen Werth, denn theils krystallisiren diese sehr leicht löslichen Salze nicht und habon gar keine charakteristischen Eigenschaften, theils aber ist bei der Oxysäure zur Lösung des Lactonringes ein sehr langes Kochen mit einem grossen Ueberschuss von Baryt erforderlich, wobei Umlagerungen stattfinden können. Noch mehr aber verlieren die Salze an Beweiskraft durch die Thatsache, 
dass sie, gleichgültig, ob aus dem Dilacton oder der Lactonsäure bereitet, nach dem Zersetzen mit Salzsäure weder das Dilacton noch die Oxysäure glatt zurïckbildeten, sondern im Wesentlichen eine nicht krystallisirende Säure lieferten.

Man kann gegen die obige Annahme den Einwurf machen, dass nach unseren bisherigen Erfahrungen bei Wasseranlagerung an doppelt gebundene Kohlenstoffatome die $\mathrm{OH}$-Gruppe in der Regel das Kohlenstoffatom bevorzugt, welches die geringstc Anzahl von Wasserstoffatomen hat, aber hier liegt, wie schon das Ausbleiben oder Zurücktreten der zweiten Lactonbindung bei einem am $\gamma$-Kohlenstoffatom gebundenen Bromatom zeigt, ein aussergewöhnlicher Fall vor, da die Reactionen sich in einem Lactonringe abspielen.

Wenn man diese Ableitung für gezwungen und deshalb für nicht sehr wahrscheinlich hält, so bleibt nur die Annahme übrig, dass das Bromatom der Bromisoterebinsäure sich einfach gegen Hydroxyl austausche. Dann käme der Oxyisoterebinsäure die Formel<smiles>CC1(O)COC(=O)C1CC(=O)O</smiles>

zu. Allein warum diese $\gamma$-Oxysäure nicht in das Dilacton überzugehen vermag, bleibt unverständlich und man ist zu der weiteren Hypothese genöthigt, dass räumliche Verhältnisse das unmöglich machen. Mir erscheint diese Annahme viel gezwungener, als die oben entwickelte.

Einfacher als bei der Dimethylaticonsäure liegen die Verhältnisse bei den beiden anderen untersuchten Aticonsäuren. Hier geben die Bromlactonsäuren neben den Dilactonen nur diejenigen Verbindungen, in welche die Dilactone selbst unter den gleichen Versuchsbedingungen weiter umgewandelt werden.

Alle drei Dilactone nämlich gehen bei längerem Kochen mit Wasser in isomere ungesättigte Lactonsäuren über, welche ich als Isaconsäuren bezeichne, weil sie verschieden von den 
Fittig, Umlagerung zweibasischer ungesättigter Säuren. 139 gleich zusammengesetzten Aconsäuren (Terebilensäure, Isobutaconsãure u. s. w.) sind, die aus den Itaconsäuren ebenso durch successive Einwirkung von Brom und Wasser gebildet werden.

Da diese Isaconsäuren durch Natriumamalgam glatt in die gesättigten Isoparaconsäuren übergeführt werden, während die länger bekannten Aconsäuren unter den gleichen Bedingungen die gewöhnlichen Paraconsäuren geben, so muss bei der Bildung der Isaconsäuren aus den Dilactonen derjenige Lactonring, welcher in den Aconsäuren und Paraconsäuren enthalten ist, geöffnet werden. Die Isaconsäuren entstehen aus den Dilactonen demnach auf folgende Weise:<smiles>[R12]C1OC(=O)C1C[Y7]#CC1OC(=O)C(CC(=O)O)C1O</smiles>

Dilacton

oder<smiles>CO[Al]1CC(CC(=O)O)C(=O)O1</smiles><smiles>C=[V]C1OC(=O)CC1CC(=O)O</smiles>

Isaconsäure.

Bei der Isoterebilensäure aus der Dimethylaticonsäure werden die Verhältnisse insofern noch complicirter, als aus dem Dilacton drei verschicdene ungesättigte Lactonsäuren mit dem gleichen Lactonringe entstehen können: 
Fittig, Umlagerung zweibasischer ungesättigter Säuren.<smiles>CC12COC(=O)C1CCOC2=O</smiles>

Isoheptodilacton<smiles>CC1(O)COC(=O)C1CC(=O)O</smiles>

umbekannte Oxylactonsäure<smiles>CC(COC(=O)CC(CC(=O)O)C(C)(COO)C(=O)OO)C(=O)OO</smiles>

Isoterebilensäure.

Die grosse Bestäudigkeit der Isoterebilensäure und der homologen Isaconsäuren macht entschieden die Formeln (2) zu den wahrscheinlichsten.

Die aus der Isoterebilensäure durch Reduction entstehende Isoterebinsäure ist in jedem Falle nach der Formel

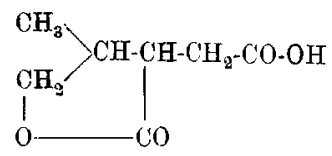

constituirt und für die aus den anderen Isaconsäuren entstehenden Isoparaconsäuren ergiebt sich ebenso die Formel<smiles>[Y2]C1CC(CC(=O)O)C(=O)O1</smiles>

Das ist die gleiche Formel, zu welcher ich für die Isopropylisoparaconsäure in Folge ihrer Bildung durch Oxydation der Isobutylbernsteinsäure gelangt bin (siehe Seite 125).

Die Isaconsäuren entstehen aus den Bromlactonsäuren aus Isobutyl- und Hexylaticonsäure aber auch schon in kalter al- 
kalischer Lösung und beim Kochen mit Wasser durch einfache Abspaltung von Bromwasserstoff. Selbstverständlich können auch so Säuren von den zwei obigen Constitutionen entstehen. Es ist indess sehr wahrscheinlich, dass nur ungesättigte Lactonsäuren von den Formeln (2) beständig sind. Die nach Formel (1) constituirten werden bei Lösung des Lactonringes, in derselbeu Weise, wie ich es bei den ungesättigten Lactonen dieser Art nachgewiesen habe, in $\gamma$-Ketonsäuren übergehen. Das findet statt bei der Hexylverbindung, wo in beiden Fällen (mit kalter Natronlauge oder mit siedendem Wasser) neben der Hexylisaconsäure eine zweibasische Säure, $\mathrm{C}_{11} \mathrm{H}_{18} \mathrm{O}_{5}$, entsteht, welche sehr wahrscheinlich Ketoheptylbernsteinsäure,

ist. $\mathrm{CH}_{3}-\left(\mathrm{CH}_{2}\right)_{4}-\mathrm{CO}-\mathrm{CH}_{2}-\mathrm{CH}-\mathrm{CH}_{2}-\mathrm{CO}-\mathrm{OH}$,

Die Ursache, dass die Bromisoterebinsäure aus der Dimethylaticonsäure sich anders verhält und beim Kochen mit Wasser weder die Isoterebilensäure noch eine Ketonsäure (welche hier übrigens gar nicht entstehen kann), sondern die mit der letzteren isomere Oxylactonsäure giebt, liegt offenbar darin, dass bei ihr die Bromwasserstoffabspaltung noch in einer dritten, der vorhin (Seite 137) besprochenen Weise stattfinden kann, und diese ist augenscheinlich die bevorzugte. Die Entstehung einer einbasischen Oxylactonsäure aus einer ungesättigten Lactonsäure von der Formel (3) Seite 140 ist aber der gleiche Vorgang, wie die Bildung einer zweibasischen Ketonsäure aus einer Säure von der Formel (1).

Man sicht aus dieser Darlegung der Resultate unserer Arbeit, dass im Einzelnen noch Manches übrig bleibt, was weiter zu untersuchen und sicherer festzustellen ist, als es bisher möglich war; aber die Hauptaufgabe, die Constitution der neuen Säuren zu ermitteln, darf für die drei aliphatischen Aticonsäuren wohl als gelöst angesehen werden. Nur durch die oben (Seite 135) aufgeführten Formeln lassen sich die beobachteten Thatsachen erklären. Die Säuren müssen structurverschieden von 
den Itaconsäuren sein. Die Annahme bloss stereoisomerer Beziehungen zwischen den Aticon- und Itaconsäuren führt, wie ich hier nicht weiter zu entwickeln brauche, auf Schritt und Tritt zu Widersprüchen mit den Thatsachen und bei der Dimethylaticonsäure ist sie überhaupt unmöglich.

Es bleibt aber die Phenylaticonsäure, bei welcher uns die obige Erklärungsweise im Stiche lässt und Structurverschiedenheit von der Phenylitaconsäure nicht angenommen werden kann. Das ist eine ausserordentlich grosse Schwierigkeit, denn es ist ganz zweifellos, dass Jemand, der von allen theoretischen Betrachtungen absieht und nur die beobachteten Thatsachen sprechen lässt, niemals auf den Gedanken kommen, oder es auch nur für annehmbar erachten wird, dass die Phenylaticonsäure einer ganz anderen Körperklasse angehöre, als die aliphatischen Aticonsäuren. Bildet sie sich doch aus der Phenylitaconsäure auf genau dieselbe Weise und sogar in gleicher relativer Menge, wie die anderen Aticonsäuren aus den entsprechenden Itaconsäuren und wird sie doch, genau wie jene, beim Kochen mit Natronlauge wieder in gleichem Maasse in die Itaconsäure zurückverwandelt. Alle Unterschiede im Schmelzpunkte, den Löslichkeitsverhältnissen und den sonstigen physikalischen Eigenschaften zwischen den aliphatischen Aticonsäuren und den isomeren Itaconsäuren finden sich in gleicher Weise zwischen der Phenylaticonsäure und der Phenylitaconsäure wieder, und anch in chemischen Verbalten zeigt sich zwischen der Phenylaticonsäure und der Dimethylaticonsäure kaum ein grösserer Unterschied, als zwischen dieser Säure und einer der beideu anderen untersuchten Aticonsäuren, oder als zwischen der Phenylitaconsäure und einer aliphatischen Itaconsäure. Nur gegen Brom verhält sich die Phenylaticonsäure in einer Beziehung anders, wie die anderen Aticonsäuren. Kleine Mengen Broms verwandeln die in Auther unter Chloroformzusatz gelöste Phenylaticonsäure im directen Sonnenlichte ebenso in die Phenylitaconsäure zurück, wie die Citraconsäuren so in die Mesaconsäuren umgewandelt werden. Bei keiner der anderen Aticon- 
säuren haben wir diese Umlagerung bewirken können, aber auch bei der Phenylaticonsäure verschwindet das freie Brom ausserordentlich rasch und die Umwandlung findet einigermassen glatt nur dann statt, wenn man die Phenylitaconsäure durch Eintragen einer Spur dieser Säure oder durch Kratzen der Gefässwände mit einem Glasstabe rasch zur Abscheidung bringt. Bei der Einwirkung von Brom im zerstreuten Tageslichte auf die Lösung der Phenylaticonsäure in Aether-Chloroform, oder besser noch in Wasser, entsteht, wie wir erst in der letzten Zeit beobachteten, auch aus ihr eine Bromlactonsäure, welche verschieden ist von den aus der Phenylitaconsäure unter den gleichen Umständen gebildeten, früher ${ }^{3}$ ) beschriebenen beiden Phenylbromparaconsäuren. Das Studium dieser Säure ist noch nicht abgeschlossen. Ich werde darüber später in einem Nachtrage zu dieser Untersuchung berichten.

Trotz dieser grossen Uebereinstimmung mit den anderen Aticonsäuren giebt es bei dem augenblicklichen Stande der Wissenschaft keinen anderen Ausweg, als die Verschiedenheit der Phenylaticonsäure von der Phenylitaconsäure auf verschiedene räumliche Lagerung zuräckzufuhren. Die hier in Betracht kommenden stereoisomeren Verhältnisse sind so einfacher Art, dass mir ein weiteres Eingehen darauf überflüssig erscheint.

Am Schlusse der Abhandlung theile ich noch einige Versuche uber das Verhalten der vier isomeren Klassen von zweibasischen Säuren bei der Oxydation mit. Diese Untersuchang wird im Augenblicke noch fortgesetzt, aber die bis jetzt erhaltenen Resultate bieten schon manches Interessante.

Nur bei einer einzigen Säure, der gewöhnlichen Itaconsäure, ist uns bisher die Oxydation ohne Spaltung des Moleküls gelungen. Ganz analog wie die einbasischen $\beta \gamma$-ungesättigten Säuren zu Oxylactonen oxydirt werden, geht sie in eine Oxylactonsäure, die Oxyparaconsäure, über:

3) Diese Annalen $\mathbf{2 5 6}, 76$. 


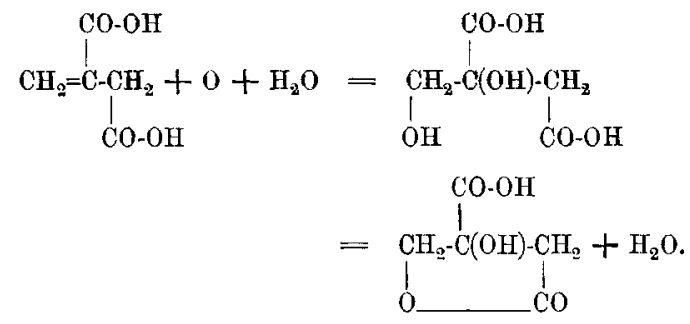

Die Isobutylitaconsäure und die Phenylitaconsäure dagegen werden in die Aldehyde, Malonsäure und Kohlensäure gespalten. Die Reaction ist leicht verständlich:

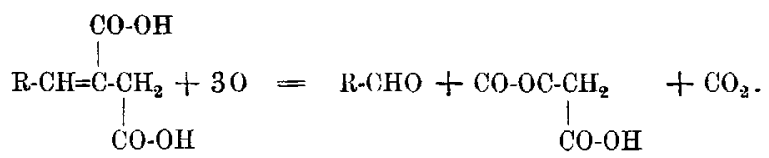

Oxalsäure entsteht dabei nicht, oder nur in geringer Menge als secundäres Product.

Ganz anders verhalten sich die Citra- und Mesaconsäuren. Beide liefern weder Aldehyde noch Malonsäure, sondern Oxalsäure und $\alpha$-Ketonsäuren, die einfache Citra- und Mesaconsäure: Brenztranbensäure; die Isobutylcitra- und -mesaconsäure: Isobutylbrenztraubensäure. Der Process verläuft nach dem Schema:

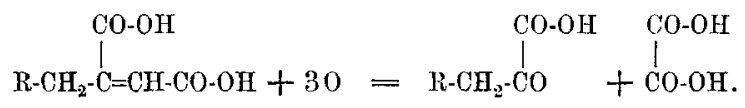

Da die $\alpha$-Ketonsäuren leicht weiter oxydirt werden, entstehen als Nebenproducte geringe Mengen der Fettsäuren mit einem Kohlenstoffatom weniger; aus der gewöhnlichen Citraund Mesaconsäure: Essigsäure, aus der Isobutylcitra- und mesaconsäure: Isocapronsäure.

Die Bildung der aliphatischen $\alpha$-Ketonsäuren auf diese Weise wird als Darstellungsmethode dieser Säuren zu verwerthen sein. Nach dem Verhalten der genau untersuchten Isobutylbrenztraubensäure zu schliessen, baben die Homologen keineswegs die Unbeständigkeit der Brenztraubensäure, sondern schliessen sich in ihren Eigenschaften eng an die bisher bekannten aromatischen $\alpha$-Ketonsäuren an. 
Von den $\Lambda$ ticonsäuren ist bis jetzt nur die Phenylaticonsäure oxydirt worden. Sie liefert die gleichen Producte, wie die Phenylitaconsäure.

Die Oxydation der aliphatischen Aticonsäuren ist im Augenblicke noch Gegenstand der Untersuchung. Ich werde die Resultate dieser Versuche später mittheilen.

\section{Ita-, Citra- und Mesaconsäure;}

von Charles Ford Langworthy.

Bei den im Folgenden beschriebenen Versuchen kam es darauf an, die drei Säuren scharf neben einander zu erkennen und möglichst quantitativ von einander zu trennen. Die Angaben, die sich in der Literatur über die Eigenschaften der Säuren und ihrer Salze finden, reichten dazu nicht aus. Wir haben deshalb die drei freien Säuren mit der grössten Sorgfalt gereinigt und mit einander verglichen, ausserdem ihre sehr charakteristischen Baryumsalze genau untersucht und auch deren so sehr verschiedene Löslichkeit in Wasser genau bestimmt.

Itaconsäure. Die reine Säure schmilzt bei $161^{\circ}$ unter theilweiser Zersetzung. Sie ist ziemlich schwer löslich in reinem Aether und fast unlöslich in Chloroform, Schwefelkohlenstoff, Benzol, Tetrachlorkohlenstoff und Ligroïn. Mit Wasserdämpfen ist sie nicht flüchtig. Die widersprechenden Angaben von Frantz ${ }^{1}$ ) sind nieht richtig. Dass er bei der Destillation im Dampfstrome ein saures Destillat erhielt, in welchem die Itaconsäure nicht direct nachgewiesen, sondern nur durch Titriren mit Natronlauge bestimmt wurde, rührt, wenn die angewandte Itaconsäure ganz rein war, nur daher, dass in einem raschen Dampfstrome, wenn nicht besondere Vorsichtsmaassregeln angewandt werden, immer Partikelchen der Flüssigkeit mit übergerissen werden. Vermeidet man diese Fehlerquelle oder

1) Wiener Monatsh. 15, 210. 